\title{
ENSEÑANZA DEL MÉTODO DE BISECCIÓN EN LA SECUNDARIA CON GEOGEBRA COMO MEDIO DIDÁCTICO
}

\author{
TEACHING OF THE BISECTION METHOD IN \\ THE SECONDARY SCHOOL WITH GEOGEBRA \\ AS A DIDACTIC MEDIA
}

\author{
Jesús David Berrio Valbuena ${ }^{1}$ \\ Sthefanie Loraine Cantillo Ortiz ${ }^{2}$ \\ Óscar Wilson Calderón Barrera ${ }^{3}$
}

Universidad del Atlántico

\section{RESUMEN}

Esta investigación tiene como objetivo estudiar el desarrollo de estrategias de los estudiantes de grado once para resolver problemas que implican el cálculo de soluciones numéricas de ecuaciones no lineales. Se plantea el diseño y ejecución de una Ingeniería Didáctica de la cual implementaremos 3 fases: La primera fase corresponde al Análisis preliminar, consistente en un análisis epistemológico del objeto matemático

1 Magister en Educación Matemática y Licenciado en Matemáticas, Docente Investigador Grupo GIHEM, Universidad del Atlántico, Correo electrónico: jberriovalbuena@ mail.uniatlantico.edu.co, ORCID: https://orcid.org/00000002-4014-5322

2 Licenciada en Matemáticas, Estudiante Grupo GIHEM, Universidad del Atlántico, Correo electrónico: slcantillo@mail.uniatlantico.edu.co, ORCID: https://orcid.org/00000002-0015-972X

3 Licenciado en Matemáticas, Estudiante Grupo GIHEM, Universidad del Atlántico, Correo electrónico: owcalderon@mail.uniatlantico.edu.co, ORCID: https://orcid. org/0000-0002-0964-6591 de estudio y el análisis de la enseñanza tradicional y sus efectos; en la segunda fase, llamada Análisis a priori, se hace una levantamiento hipotético refinado de las estrategias que pueden surgir durante la aplicación de las situaciones didácticas; a la aplicación de las situaciones didácticas en el aula y el registro audiovisual; y en la última fase, la de análisis a posteriori y evaluación, se practicarán unos análisis locales, con las estrategias de solución de problemas de cada estudiante y un análisis global para establecer generalidades entre las estrategias evidenciadas. El impacto real de la intervención didáctica en esta investigación se mide en términos de la construcción del método de bisección para calcular soluciones aproximadas de ecuaciones no lineales. Los resultados muestran que se lograron identificar y analizar algunas de las estrategias o técnicas 
utilizadas por los estudiantes, las cuales se evidenciaron en la realización de las actividades tanto en el estudiante A como en el estudiante $B$, todas estas contribuyendo en la formación del concepto del método de bisección.

\section{PALABRAS CLAVES}

Método de bisección, GeoGebra, Situaciones didácticas, ecuaciones no lineales, Ingeniería didáctica.

\section{ABSTRACT}

This research aims to study the development of strategies of eleventh grade students to solve problems that involve the calculation of numerical solutions of non-linear equations. The design and execution of a Didactic Engineering of which 3 phases are implemented is proposed: The first phase corresponds to the Preliminary Analysis, consisting of an epistemological analysis of the mathematical object of study and the analysis of traditional teaching and its effects; In the second phase, called A priori analysis, a refined hypothetical survey is made of the strategies that may arise during the application of the didactic situations; to the application of didactic situations in the classroom and the audiovisual record; and in the last phase, that of a posteriori analysis and evaluation, local analyzes will be practiced, with the problem-solving strategies of each student and a global analysis to establish generalities between the strategies evidenced. The real impact of the didactic intervention in this research is measured in terms of the construction of the bisection method to calculate approximate solutions of non-linear equations. The results show that it was possible to identify and analyze some of the strategies or techniques used by the students, which were evidenced in the performance of the activities in both student $A(E A)$ and student $B(E B)$, all of which contribute in the formation of the concept of the bisection method.

\section{KEYWORDS}

Bisection methods, Geogebra, Didactic situations, Non-linear equations, Didactic engineering.

\section{RESUMO}

Esta pesquisa tem como objetivo estudar o desenvolvimento de estratégias de alunos do $11^{\circ}$ ano para a resolução de problemas que envolvam o cálculo de soluções numéricas de equações não lineares. Propõe-se o desenho e execução de uma Engenharia Didática da qual implementaremos 3 fases: A primeira fase corresponde à Análise Preliminar, consistindo na análise epistemológica do objeto de estudo matemático e na análise do ensino tradicional e seus efeitos; $\mathrm{Na}$ segunda fase, chamada de Análise A priori, é feito um levantamento hipotético apurado das estratégias que podem surgir durante a aplicação das situações didáticas; à aplicação de situações didáticas em sala de aula e no registro audiovisual; e na última fase, a de análise e avaliação a posteriori, serão praticadas análises locais, com as estratégias de resolução de problemas de cada aluno e uma análise global para estabelecer generalidades entre as estratégias evidenciadas. O real impacto da intervenção didática nesta pesquisa é medido em termos da construção do método da bissecção para calcular soluções aproximadas de equações não lineares. Os resultados mostram que foi possível identificar e analisar algumas das estratégias ou técnicas utilizadas pelos alunos, as quais foram evidenciadas no desempenho das atividades tanto do aluno $A(E A)$ quanto do aluno $B(E B)$, todos contribuindo na a formação do conceito de método de bissecção.

\section{PALAVRAS-CHAVE}

Método de bissecção, GeoGebra, Situações didáticas, Equações não lineares, Engenharia Didática. 


\section{INTRODUCCIÓN}

El presente proyecto de investigación surge de la necesidad de atender las recomendaciones y conclusiones de la investigación de Ochoa y Osorio (2017), que indican que aunque el concepto de método de bisección no está incluido en los lineamientos curriculares, ni en los estándares básicos de competencias del Ministerio de Educación Nacional (MEN), es posible lograr generar en el aula una situación que propicie su enseñanza y aprendizaje en una aula de educación secundaria (ValbuenaDuarte, De La Hoz y Berrío-Valbuena, 2021). La población de esta investigación son los estudiantes de undécimo grado de la Institución Educativa Distrital de Formación Integral y la muestra fueron dos estudiantes elegidos de manera no probabilística. Las técnicas e instrumentos utilizados en la investigación fueron la entrevista clínica, videograbaciones y transcripciones.

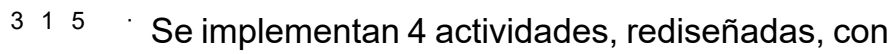
el fin de lograr un aprendizaje por adaptación. Estas actividades separaron el concepto de bisección, en conceptos como: continuidad, punto medio, teorema de Bolzano y por último el método de bisección, con el fin de reforzar los conceptos previos de los estudiantes y facilitar el aprendizaje del último.

El marco teórico de esta investigación está soportado en la Teoría de las Situaciones
Didácticas (TSD) de Brousseau (1997; 2007), precisamente las situaciones a-didácticas y el aprendizaje por adaptación, pues se propone la aplicación de actividades a los estudiantes con la intención de que estos puedan resolverlas sin la intervención del docente en la construcción de estrategias de solución o del concepto como tal.

Según Brousseau (1997; 2007) y Acosta (2010), en las Situaciones A-didácticas el aprendizaje por adaptación se da esencialmente en la interacción entre el medio y el sujeto; este medio cumple una función dependiendo de la fase en la que se encuentre (acción, retroacción y validación): El sujeto parte de una intención, de una meta a alcanzar, por lo cual realiza una acción sobre el medio, el medio reacciona a esta acción, a esto se le llama en la TSD una retroacción, luego el sujeto hace un interpretación de la retracción mostrada por el medio y finalmente el sujeto valida su acción de acuerdo con la interpretación que hace de las retroacciones del medio. Esta validación puede tomar dos valoraciones: Cuando la acción realizada le permite alcanzar su intención la validación es positiva, en cuyo caso refuerza esta acción, es decir la repetirá con mayor frecuencia cuando quiera alcanzar esa intención. Cuando la acción realizada no le permite alcanzar su intención la validación es negativa y produce una modificación de la acción, iniciando un nuevo ciclo acciónretroacción-validación, el medio puede ser material o virtual, ver figura 1 .

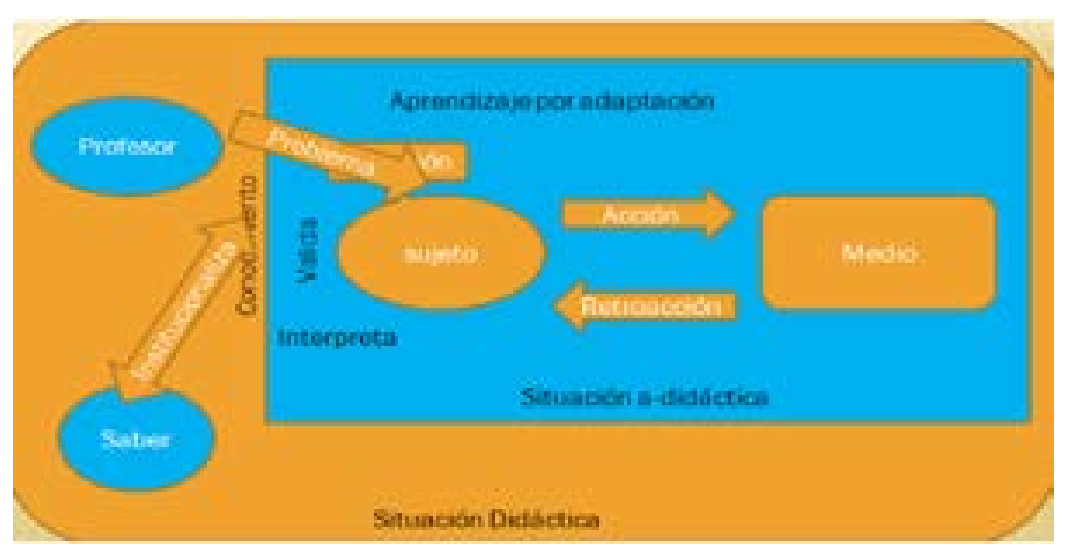

Figura 1. Esquema de la Teoría de las Situaciones Didácticas (Tomada de Acosta (2010)) 
El objetivo de esta investigación es que los estudiantes puedan construir el método de bisección, para ello se utilizan las actividades antes mencionadas, dado que al enfrentar a los estudiantes (sujetos) ante situaciones problemáticas donde deben cumplir una tarea, éstos buscarán la manera de tener éxito, construyendo estrategias que les permitan solucionar dicha situación, en medio de un contexto con el que interactúan, demostrando así, que se logra una adaptación. Se busca evidenciar que, al finalizar el proceso, los estudiantes son capaces de reconocer la existencia de raíces o soluciones de una ecuación no lineal y qué pasos deben seguir para dar certeza de ello.

La metodología se basa en la realización de un análisis a priori, de cada tarea y actividad, para evaluar las posibles, acciones, retroacciones e interpretaciones del estudiante, posteriormente se pretende contrastar el análisis a priori con el análisis a posteriori y llegar a una conclusión, a fin de validar si el objetivo de la tarea y la actividad fue cumplido o no. Es necesario aclarar que las situaciones mediadas en Geogebra propuestas por Ochoa y Osorio (2017) han sido rediseñadas, como se especifica en sus recomendaciones, y por esto es necesario el rediseño del análisis a priori.

Luego de observar y estudiar las acciones y estrategias utilizadas por los estudiantes y al tener en cuenta los análisis a posteriori se llegó a la conclusión que los estudiantes lograron una apropiación sobre cómo identificar la existencia de una raíz de una función en un intervalo dado, uno de ellos logró alcanzar los objetivos de manera satisfactoria, apropiándose del paso a paso para la implementación del método de bisección y la identificación de la raíz utilizando el teorema de Bolzano (Calderón y Cantillo, 2018).

\section{MATERIALES Y MÉTODOS}

El enfoque de esta investigación es cualitativo, apoyado en la estrategia clínica, en razón de que facilita la construcción de diseños investigativos del mundo empírico, permitiendo a los investigadores conectar sus supuestos teóricos con formas específicas de obtener información para analizarla (Camargo, 2019). Se implementó este método dado que según Cervantes-Barraza, Berrío-Valbuena, Contreras-Vásquez y Martínez-Fontalvo (2021) es un proceso que consiste en someter a un objeto o grupo de individuos (los estudiantes de undécimo grado), a determinadas condiciones, estímulos o tratamiento (las situaciones a-didácticas mediadas con Geogebra), para observar los efectos o reacciones que se producen en los estudiantes, pues se pretende mostrar la relación que hay entre causa-efecto, más explícitamente como aprendizajes a priores y a posteriores.

De acuerdo con la teoría de Transposición Didáctica (Chevallard, 1998) y la Teoría de Situaciones Didácticas (Brousseau, 1997; 2007), se concuerda en que el diseño de esta investigación está fundamentado en la Ingeniería Didáctica, en esta analizaremos la importancia de realizar un análisis a priori y un análisis a posteriori.

La Ingeniería Didáctica como metodología para la investigación planteada, permite llevar a cabo un análisis en el acercamiento de los estudiantes a la aproximación del método de bisección, está apoyada de las diferentes fases propuestas por Artigue, Douady y Moreno (1995).

Fase 1. Análisis preliminar: en esta fase se analizará la desventaja de la enseñanza de las ecuaciones no lineales por medio del método tradicional y a partir de esto se rediseñarán las actividades (situaciones a-didácticas) con el fin de abarcar los objetivos propuestos en el primer capítulo. 
Fase 2. Fase de concepción y análisis a priori de las situaciones didácticas: en esta fase se analizará todos aquellos factores que pueden intervenir en el estudio o en sus resultados. Se examinarán las características de las actividades, con el fin de inferir posibles respuestas y las estrategias que podrían utilizar los estudiantes al desarrollar las actividades antes mencionadas.

Fase 3. Experimentación: en esta tercera fase se procederá a implementar las actividades propuestas y se dará cabida a la recolección de datos respectivamente, esto sin dejar de lado la verificación de los pasos ejecutados por los estudiantes, para así tener una mejor visión de cómo lo interpretan y si son capaces de sustentar lo realizado.

Fase 4. Análisis a posteriori y evaluación: en esta última fase de la Ingeniería Didáctica, se procederá a realizar un análisis detallado de los resultados obtenidos en la fase anterior, para que de esta forma se realice un contraste entre nuestras expectativas (descritas en la fase número dos) y los resultados reales (descritas y mostradas en la fase número tres).

\section{ENFOQUE CLÍNICO}

Desde la perspectiva de Hunting (1997), Zazkis y Hazzan (1999) y Clement (2000) quienes manifiestan que este tipo de enfoque permite al investigador ampliar su experiencia acerca de cómo trabaja matemáticamente la mente del individuo, El objetivo es transformar las ideas del estudiante en material oral y gesticular que mediante las videograbaciones podrá ser capturada para analizarse. Para esta investigación, se utilizaron técnicas e instrumentos precisos que permitieron la recolección y el análisis de los datos obtenidos, estos fueron:

- La entrevista clínica: esta técnica permite estudiar la forma original de las estructuras de conocimiento y los procesos de razonamiento de cada individuo. Estas entrevistas permiten recopilar y analizar datos sobre los procesos mentales en el plano de las ideas auténticas de un sujeto y sus significados, además de exponer las estructuras y procesos ocultos en el pensamiento del sujeto que no pueden ser detectados con otras técnicas (Hunting, 1997; Clement, 2000).

- Observación: es una técnica que consiste en visualizar o captar mediante la vista, en forma sistemática, cualquier hecho, fenómeno o situación que se produzca en la naturaleza o en la sociedad, en función de unos objetivos de investigación preestablecidos, se realiza una observación de manera neutral sin involucrarse en el medio o realidad en la que se realiza el estudio (Arias, 2012).

- Grabaciones en vídeo: Los métodos audiovisuales según Schnettler y Baer (2009) y García (2011) constituyen una manera de abrir nuevos caminos en la formas de acercarse a sus objetos de estudio y explorar realidades. En esta investigación, para poner de manifiesto qué hace y qué dice el estudiante mientras resuelve los problemas propuestos se usan videograbaciones. Los videos se usarán para elaborar las transcripciones de los episodios que los investigadores consideren susceptibles de ser analizados para obtener sustento que permita hablar acerca de las estrategias de resolución de los problemas propuestos (Berrío, Acosta y Fiallo, 2013; Berrío, 2016).

- Transcripción: la transcripción es un registro escrito de una entrevista, narración, anotación y otros elementos similares. La cual refleja el lenguaje verbal, no verbal y contextual de los datos (Hernández, Fernández y Batista, 2014). Las transcripciones convierten las estrategias de solución creadas por los estudiantes en material documental necesario para la interpretación e análisis de 
la información, asimismo permite registrar mediante documentación las experiencias y hechos relevantes para el desarrollo de la investigación (Sánchez y Revuelta, 2005).

\section{DESCRICPCIÓN DE LAS ACTIVIDADES Y ANÁLISIS A PRIORI}

Teniendo en cuenta la metodología planteada en esta investigación, a continuación, se describen las actividades rediseñadas y su objetivo de acuerdo a las conclusiones de Osorio y Ochoa (2017) y a las indicaciones de Allan, Parra y Martins (2017).

El análisis a priori de esta investigación inicia con la identificación de los conceptos de método de bisección (en sí mismo), el concepto de continuidad de una función, definición de punto medio en un intervalo y el teorema de Bolzano como los saberes teóricos previos asociados al Método de Bisección como estrategia para la resolución de ecuaciones no lineales.

Método de bisección: Este es uno de los métodos más sencillos y de fácil intuición, para resolver ecuaciones en una variable. Se basa en el Teorema de los Valores Intermedios, el cual establece que toda función continua $f$ en un intervalo cerrado $[a, b](f \in C[a, b])$

toma todos los valores que se hallan entre $y$. Esto es, que todo valor entre $f(a)$ y $f(b)$,

$\mathrm{y}$ es la imagen de al menos un valor en el intervalo $[a, b]$.

(Mora, 2018; Canale y Chapra, 2014)

Es decir, que en si en el intervalo $[a, b]$. hay un cero de $f$. Calculamos el punto medio $(m)$ de dicho intervalo, para luego obtener la imagen de $m$, en caso de que la imagen de sea igual a cero, hemos encontrado entonces la solución buscada. (Mora, 2018)

Este método hace uso de la aplicación del teorema de Bolzano n-veces, donde el número de veces es determinado por la precisión con la que se quiera la solución buscada, siempre y cuando la función sobre la cual se está aplicando este proceso sea continua en el intervalo de búsqueda de la solución. Este proceso iterativo comienza al dividir el intervalo de búsqueda de la solución en 2 sub-intervalos de igual tamaño (se halla el punto medio del intervalo), reteniendo el sub-intervalo en donde las imágenes de $f$ cambien de signo para conservar al menos una raíz o cero (Teorema de Bolzano), y repetir el proceso varias veces (Ochoa y Osorio, 2017).

Con base en las anteriores apreciaciones se considera la aplicación de las siguientes actividades. En cada una de ella se plantea desarrollar los saberes previos y la conjugación de estos en la construcción heurística del método de bisección como la estrategia de solución de ecuaciones no lineales.

Actividad 1 (El termómetro): Esta actividad está compuesta por la construcción de una simulación de un termómetro en GeoGebra (ver tabla 1) que puede ser manipulado mediante una perilla, con ella el estudiante puede hacer las respectivas variaciones establecidas en el desarrollo de las tareas propuestas. El termómetro antes mencionado estará dado en grados Celsius $\left({ }^{\circ} \mathrm{C}\right)$ y cambiará de color de azul a rojo, al pasar de una temperatura negativa a positiva o viceversa.

En la tarea 1 y 2 , se le pide al estudiante que gire la perilla desde la posición inicial $\left(-50^{\circ} \mathrm{C}\right)$ hacia la medición de $30^{\circ} \mathrm{C}$, luego desde esa posición hasta la medición de $-10^{\circ} \mathrm{C}$, y luego que la lleve hasta la medición de $40^{\circ} \mathrm{C}$. Aunado a esto se le pregunta al estudiante ¿Qué observó en el medidor de temperatura del termómetro, cuando realizó los cambios de temperatura sugeridos? y ¿Dónde se produce el cambio en el medidor de temperatura del termómetro observado? Teniendo como hipótesis que el estudiante logre reconocer que existe un cambio de color en el termómetro y que éste depende de las temperaturas que se señalen con la perilla, 
además que dicho cambio se produce al pasar $0^{\circ} \mathrm{C}$.

En la tarea 3, se le proponen las mismas acciones de las tareas anteriores pero con la condición de que el medidor de temperatura no pase por $0^{\circ} \mathrm{C}$. La retroacción recibida por la simulación es que no es posible girar la perilla de un temperatura negativa a positiva o viceversa sin pasar por $0^{\circ} \mathrm{C}$, por lo que la hipótesis a priori es que el estudiante interpretará esto concluyendo que no es posible cumplir con la tarea propuesta

Actividad 2 (Continuidad): Esta actividad se encuentra conformada por una serie de cinco gráfica y en cada una de ellas se resuelve la misma tarea (ver tabla 1) y 2 preguntas a las que el estudiante debe dar respuesta, esto basándose en lo observado en las gráficas. Para la visualización de cada una de ellas como se le indica al inicio de la actividad, el estudiante deberá hacer clic sostenido y arrastrar el deslizador negro que se encuentra en la parte superior. El objetivo de cada una de las tareas es que el estudiante lleve el punto $\mathrm{N}$ ubicado sobre la función dada, desde el punto $Q$ hasta $\mathrm{P}$, si es posible. Cada tarea estará acompañada de la pregunta ¿Lo lograste?, en donde deberá escoger una sola respuesta y justificarla, si es así porqué considera el que sea posible y si no, cuál es la dificultad por la cual el estudiante no puede realizar la tarea.

El objetivo de esta actividad es que el estudiante, de acuerdo al comportamiento de la función, puede o no realizar las tareas estipuladas. Es por esto que en las últimas tareas se les pregunta a los estudiantes en qué graficas pudo realizar la actividad con éxito, en cuáles no y por qué. En el levantamiento hipotético previo se asegura que si las funciones no tienen saltos, huecos o asíntotas el estudiante realizará la tarea fácilmente. De lo contrario, no podrá realizar la tarea e identificará las mencionadas características de la curva como los factores que le impiden realizarla.
Actividad 3 (Teorema de Bolzano: Una raíz): Esta actividad está conformada por una serie de cuatro gráficas de funciones y dos preguntas (ver tabla 1), teniendo en cuenta que $P=(a, f(a)) Q=(b, f(b)) ; y C=(x, f(x))$ un punto sobre la función. La tarea del estudiante consiste en analizar cómo influye en la gráfica las variaciones en las componentes (en especial en los signos) de los puntos $P$, $Q$ y $C$ antes mencionados, qué cambios se producen al momento mover el punto $\mathrm{C}$ y qué conclusiones se pueden sacar a partir de ello. Todo esto con el fin de que el estudiante tenga el primer acercamiento sobre lo que se plantea en el teorema de Bolzano sobre continuidad.

En la actividad el estudiante encontrará que el punto $\mathrm{P}$ está por arriba del eje $x$ y el punto $\mathrm{Q}$ está por debajo. El punto $\mathrm{C}$ y su coordenada $f(x)$ cambian de color al momento de pasar por el eje $x$. El estudiante concluirá que las componentes de $P$ y $Q$ son fijas y que las imágenes de $P$ y $Q$, son de signos opuestos. Mientras que el punto $C$ en su desplazamiento de $P$ hasta $Q$ obligatoriamente pasa por el eje $x$.

Actividad 4 (Teorema de Bolzano: Más de una raíz): Esta última actividad está conformada por la Actividad 4.1: Más de una raíz positiva y la Actividad 4.2: Más de una raíz negativa (ver tabla 1), cada una de ellas con una serie de puntos que pertenecen a una misma función $f(x)$, dicha función se puede trazar sin levantar el lápiz del papel, y no estará visible para el estudiante, hasta que este finalice el paso a paso de esta actividad. A medida que se generan los demás puntos que están dentro del intervalo inicialmente dado, se pueden apreciar los nuevos intervalos que van cortando al original, igualmente se denotan las componentes de los puntos en la función.

Antes de mostrar la naturaleza de la función, se presenta una pregunta, en la cual se pone en práctica el análisis que el estudiante realizó 
mientras desarrollaba en esta actividad, pues relacionará, la ubicación de los puntos acompañado de los posibles signos de las componentes de éstos. Se espera que llegue la conclusión que en el último intervalo los puntos se encuentran, en diferentes cuadrantes y además de esto sus imágenes poseen signos contrarios, lo que lo llevará a notar que es necesario que pase por el eje x para que se "unan" y por este pase la función, lo que lo aproximará al teorema de Bolzano.

Termómetro

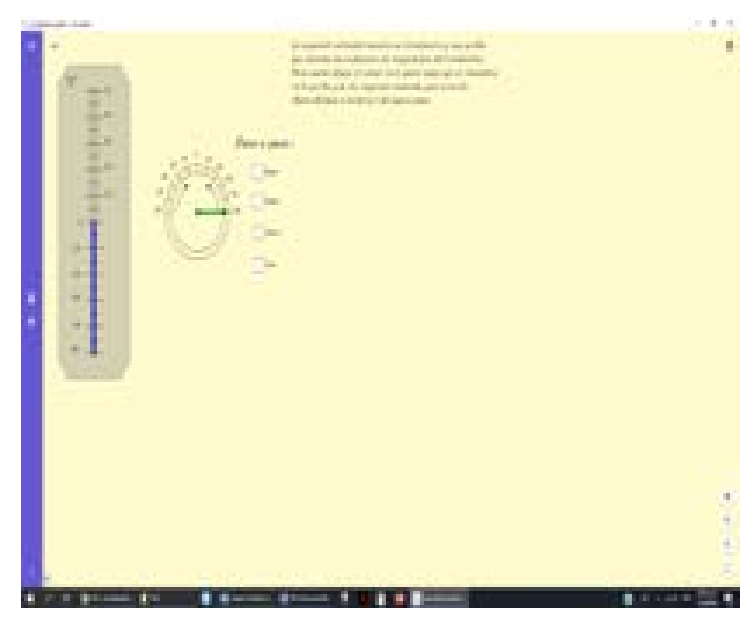

Teorema de Bolzano: una raíz

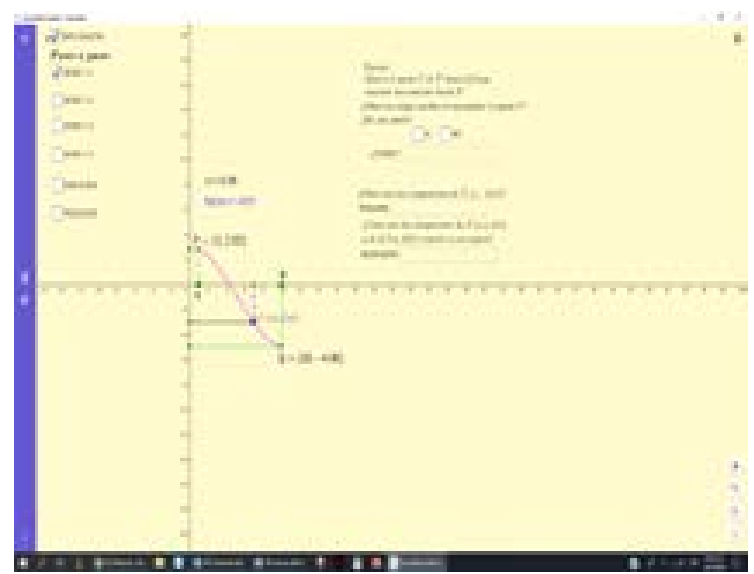

Continuidad

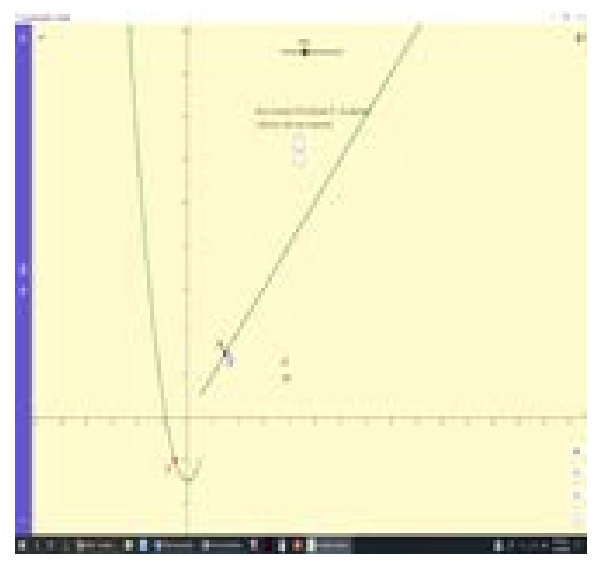

Teorema de Bolzano: más de una raíz

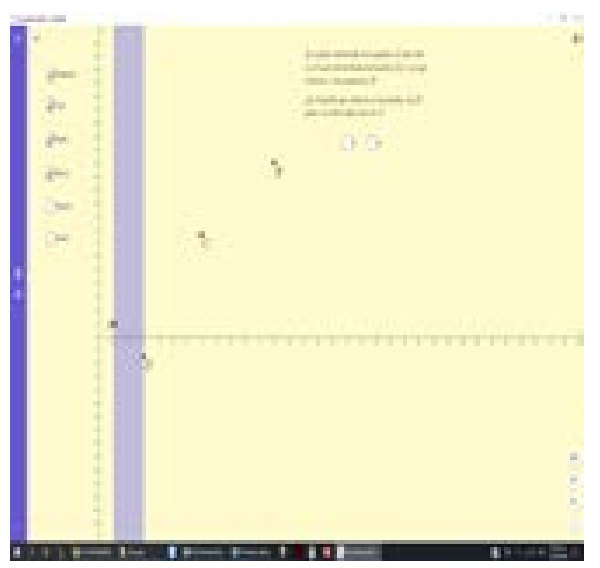

Tabla 1. Imágenes de las actividades rediseñadas y aplicadas.

\section{ANÁLISIS A POSTERIORI Y RESULTADOS}

Los análisis presentados a continuación tienen como objetivo explicitar los procesos de razonamiento detectados, las estrategias de solución propuestas y las acciones realizadas en comparación a los datos previstos en el análisis a priori.

Se usan extractos de las transcripciones realizadas a partir de las videograbaciones, para exponer las situaciones que se consideran susceptibles de análisis y revisión. La letra 
en negrilla señala lo que el estudiante o el investigador dicen, lo contrario señala lo que hacen en el desarrollo de la actividad.

\section{Actividad 1}

Tarea 1. Desde el análisis a priori se propuso que los estudiantes, al momento de desplazar la flecha de la perilla de una medición de temperatura que vaya de una medida negativa a positiva o viceversa, observarían e identificarían que hay un cambio de color en el medidor de la temperatura al momento de realizar dichos cambios.

[1] EstA: Mueve la perilla hacia las temperaturas sugeridas en la tarea e intenta mover las flechas que indican el movimiento de la perilla. ¿Esto se puede mover? (Señalando con el puntero las flechas que indican el movimiento de la perilla)

[2] Inv: No. ¿Qué observas en el medidor de temperatura del termómetro, cuando realizas los cambios de temperatura sugeridos?

[3] Est A: Que va cambiando acorde a la perilla, si acá es cuarenta (señala la perilla) acá va a ser cuarenta (señala el medidor de temperatura), si es cero (señala la perilla) acá es cero (señala el medidor de temperatura) y va cambiando el color dependiendo a la temperatura, si es arriba va a ser naranja o rojo y si es abajo es azul.

En el análisis a priori se previó que los estudiantes, al momento de desplazar la flecha de la perilla de una medición de temperatura que vaya de una medida negativa a positiva o viceversa, observarían e identificarían que hay un cambio de color en el medidor de la temperatura al momento de realizar dichos cambios.

El estudiante realiza las tareas propuestas moviendo la perilla hacia las temperaturas sugeridas, lo que muestra la acción prevista [1]. Luego, al leer la pregunta ¿Qué observo...? [2], el estudiante de manera casi inmediata responde que al mover la perilla cambia el color en el medidor de temperatura del termómetro y lo asocia al signo de la temperatura, esto evidencia la retroacción que el medio proporcionó [3]; el estudiante logra validar la acción como se evidencia en [3].

Tarea 2. En el análisis a priori se estableció que por medio de la observación, los estudiantes responderían que es sobre el punto cero donde cambia el color en el capilar del termómetro.

[1] Est A: El estudiante mueve la perilla hacia las temperaturas indicadas en la tarea.

\section{[2] Inv: ¿Dónde se produce el cambio en el medidor de temperatura del termómetro?}

[3] Est A: Cuando este llega a cero. Escribe en la casilla de entrada, "cuando este llega a el valor cero".

El estudiante mueve la perilla hacia la temperatura indicada, evidenciando la acción pronosticada en el análisis a priori [1], en [3] se muestra la retroacción y a su vez la validación ya que la cuando la perilla pasa por cero, el medidor de temperatura cambia de color permitiendo al estudiante identificar donde se produce el cambio.

Tarea 3. En el análisis a priori se esperaba los estudiantes al no poder realizar la tarea pedida, lleguen a la conclusión que para realizar esta tarea necesariamente se debe pasar por el cero.

[1] Est B: El estudiante intenta hacer el cambio sugerido de temperatura, teniendo en cuenta la restricción. ¿Llévelo a 20 sin pasar por el cero?, ¡No se puede!, ¡Es imposible! Marca en la casilla de control "NO" 
[2] Al responder no, el medio le pide que justifique la respuesta dada.

[3] Est B: Como es un número negativo para llevarlo a positivo es necesario pasar por el cero. Escribe en la casilla disponible para responder, "para pasar desde un número negativo (-40) a uno positivo (20) este necesariamente debe pasar por el valor cero.

[4] Inv: ¿Puede realizar el cambio de temperatura teniendo en cuenta la restricción?

[5] Est B: Intenta hacer el cambio sugerido de temperatura, teniendo en cuanta la restricción, pero no logra hacer los cambios sugeridos. Marca en la casilla de control "NO" (Respuesta).

[6] Al responder no, el medio le pide que justifique la respuesta dada.

\section{$\begin{array}{llll}3 & 2 & 2 & \text { [7] Est B }\end{array}$}

\section{[8] 2: ¿Puedo copiar y pegar?, es la misma} razón...

En [1] y [5] se puede verificar la acción prevista en el análisis a priori. El estudiante mueve la perilla hacia las temperaturas sugeridas y no logra desarrollar la tarea asignada, la retroacción que recibe se evidencia en [1]. El estudiante concluye acertadamente que no puede mover la perilla de un valor negativo a positivo sin pasar por el cero [3]. En [2] y [6] se presentan dos momentos de retroacción automatizada del medio; el estudiante debe justificar la respuesta negativa que recibe como reacción del medio que no le permite cumplir con la tarea designada.

Aunque en el análisis a priori no se contempló que el estudiante usara la misma estrategia que le permitió realizar la primera tarea como la estrategia que le permitiría desarrollar la segunda tarea [5] y [7], es importante mencionar que este tipo de situaciones permiten suponer un alto control sobre la validez de sus acciones.

\section{Actividad 2}

En las tareas propuestas, por tener acciones comunes, se plantea que en cada una de las gráficas el estudiante trate de arrastrar el punto $\mathrm{N}$ desde el punto $\mathrm{Q}$ hasta el punto $\mathrm{P}, \mathrm{y}$ posteriormente responda si logra realizar la tarea.

Tarea 1, gráfica 1. Se previó en el análisis a priori que el estudiante al mover el punto $\mathrm{N}$ de $\mathrm{Q}$ hasta P lograría hacerlo a lo largo de la función sin encontrar interrupciones.

[1] Est B: Mueve el punto $N$ desde $Q$ hasta $P$, logra hacerlo

[2] El medio le pregunta ¿Lo lograste?

[3] Est B: Marca en la casilla de control "SI" (Respuesta).

Se evidencia en [1] la acción y la retroacción que se pronostica al mover el punto $\mathrm{N}$ de $\mathrm{Q}$ hasta $P$ y ver que este punto logra moverse sin ninguno tipo de interrupción y se valida la acción al momento de que el estudiante afirma que si logra mover el punto $\mathrm{N}$ de $\mathrm{Q}$ hasta $\mathrm{P}$ [2].

Tarea 1, gráfica 2. En el levantamiento hipotético de las acciones se planteó que el estudiante no podría llevar el punto $\mathrm{N}$ de $\mathrm{Q}$ hasta $\mathrm{P}$ dado que a lo largo de la función encontraría una asíntota y $Q$ y $P$ estarían en partes separadas de la curva.

[1] Est A: Mueve el punto $N$ desde $Q$ hasta $P$, no logra hacerlo. Pues cuando intenta pasar el punto por la asíntota, este se desaparece.

[2] Inv: ¿Dónde está N?

[3] Est A: ¡Ya se perdió!

[4] El medio le pregunta ¿Lo lograste? 
[5] Est A: Marca en la casilla de control "NO" (Respuesta).

En [1] es posible identificar la acción que se pronosticó. El estudiante mueve el punto $\mathrm{N}$ de $Q$ hasta $P$ sin lograr llevarlo de uno hasta el otro dado que los puntos estaban en partes de las función que no estaban unidas; la retroacción se evidencia en [2] y [3], ya que el punto $N$ no se pudo mover de $Q$ hasta $P$ y en [5] se observa la validación negativa sobre las acción propuesta por el estudiante para resolver la tarea.

En las gráficas 3 y 4 se presentó el desarrollo análogo por los estudiantes, salvo que en estos casos las acciones recibían una validación negativa dado que la gráfica 3 presentaba un salto y en la gráfica 4 había un hueco. Para la gráfica 5, el comportamiento fue similar al de la gráfica 1 dado que era una función continua. Es importante destacar que aunque las validaciones son negativas (en las gráficas 2, 3 y 4) cumplen con el objetivo de la actividad, que no es más que el de la identificación, mediante heurísticas, de las características de las funciones continuas y no continuas.

Finalmente, se le pregunta al estudiante

[1] Inv: ¿En cuál gráfica lograste hacer lo que se te indicó?

[2] Est A: ¿Puedo seleccionar varias?

[3] Inv: ¡Sí!

[4] Est A: Creo que es la primera y la última, ¿Verdad? (se regresa a las tareas anteriores a ratificar). La primera y quinta.

[5] Inv: ¿Cuál crees que es la razón de lograrlo?

[6] Est A: La línea por la que pasaba el punto $N$, estaba unida o era la misma en la que estaba el punto $P$, por lo tanto si podía llevarlo (el punto $\mathrm{N}$ hasta $\mathrm{P}$ ), mientras que en las otras gráficas esto no se cumplía.
Cuando el estudiante pregunta si puede escoger varias opciones permite suponer que ha identificado características comunes en las distintas gráficas presentadas. Esto es un indicio de que ha logrado clasificar las gráficas según le es posible cumplir o no con la tarea propuesta.

\section{Actividad 3}

Se espera que los estudiantes al momento de arrastrar el punto $C$ desde $P$ hasta $Q$, identifiquen que hay un cambio de color de azul a rojo o viceversa en el punto $C$ y en sus respectivos componentes x y $f(x)$ y cuando este pasa por el eje $x$, para luego asociar este cambio con los signos que toma las componentes $f(a)$ y $f(b)$ y de los puntos $P$ y $Q$ respectivamente, es decir que este cambio sucede siempre y cuando los signos de y sean diferentes.

Cuando el estudiante mueve el punto $C$ como se le indica, de $Q$ hasta $P$ y viceversa en repetidas ocasiones, verifica la acción prevista en los análisis a priori, luego el medio le pregunta si se produce algún cambio en el punto $\mathrm{C}$ y donde, a lo que el estudiante responde afirmativamente la primera interrogante y en la segunda interrogante señala que el cambio se produce en las valores de " $x$ " $y$ " $y$ " del punto $C$ al momento de pasar por el eje , para lo cual esta retroacción que le proporciono el medio no estaba prevista en el análisis a priori, puesto que en este se pronosticó que el estudiante al realizar la tarea, identificaría el cambio de color que se da en la coordenada $f(x)$, sin embargo se esperaba que el estudiante luego de identificar el cambio de color lo asociara al cambio de signo en la componente en " $y$ " del punto $C$ al pasar por el eje $x$, lo cual se logró aún sin estar previsto de este modo.

En el análisis a priori se espera que los estudiantes por medio de lo observado y lo analizado en las series anteriores, argumenten que el cambio de signo de las componentes $f(a)$ y $f(b)$ y es lo que determina el cambio de color 
en la componente $f(x)$ del Punto C. para luego así partiendo de este hecho los estudiantes concluyan que cuando y poseen signos diferentes, la gráfica que pasa por estos puntos debe pasar por el eje $x$.

En la intervención se evidencia que el estudiante no logra identificar que el cambio de signo de las componentes $f(a)$ y $f(b)$ es lo que determina el cambio de color en la componente $f(x)$ del Punto $\mathrm{C}$, sin embargo concluyó que si $f(a)$ y $f(b)$ tienen signos diferentes la gráfica obligatoriamente va a pasar por cero, o sea se intersectara con el eje $x$.

\section{Actividad 4}

Esta actividad se encuentra dividida en dos actividades las cuales son: Actividad 4.1 y la Actividad 4.2 que están conformadas por una serie cada una, las cuales poseen puntos que pertenecen a una misma función $f(x)$ donde está se puede trazar sin levantar el lápiz del papel. Adicionalmente, Identificar sobre que intervalo se tiene la certeza, que la función continua que pasa por los puntos mostrados intersecte al eje $\mathrm{x}$.

Tarea 4.1. En el análisis a priori se prevé que, por medio de la observación y teniendo en cuenta las conclusiones a las que llego en la actividad anterior, los estudiantes consideren que la gráfica de la función que pasa por los puntos $A$ y $B$ no pasa por el eje, puesto que no hay cambio de signo en los valores de las imágenes de dichos puntos.

[1] Inv: ¿La función que contiene a los puntos A y $B$ pasa o se intersecta con el eje $x$ ?

[2] Est A: Observa la región sombreada donde se encuentra definida la función y hace un movimiento con el mouse sobre la región, de forma parabólica, haciendo énfasis en que puede pasar la función varias veces sobre el eje $\mathrm{x}$ en esa región, posteriormente responde.
[3] Est A: Pues sí, sí, ¿Me explico?, sí, porque si me dicen que la región sombreada corresponde al intervalo donde está definida la función $f(x)$ eso significa que en cualquier parte de la región azulita (sombreada) puede pasar la función, por la tanto puede ser que a baje y después suba, por ende si puede pasar por el eje $\mathbf{x}$.

El estudiante analizó y observó la ubicación de los puntos $A$ y $B$, esto deja visto la acción planteada en el análisis a priori [2]. De esta forma identificó que la gráfica de la función que pasa por A y B puede pasar por el eje varias veces, haciendo un movimiento con el puntero de forma parabólica sobre la región donde está definida la función [2]. Esta interpretación de la retroacción no se previó en el análisis a priori, pero igualmente deja validar la acción que el estudiante realizó, ya que llegó de manera directa a la interpretación que se esperaba.

Con respecto a la identificación de los intervalos que garantizan la intercepción de la función con el eje, se planteó en el análisis a priori, que mediante la visualización de los puntos medios que se van generando a partir de un intervalo inicial, los estudiantes identifican que para uno de los dos nuevos intervalos que se van formando, se sigue conservando la intercepción entre la gráfica y el eje. Se logró evidenciar que la actividad cumplió el objetivo a cabalidad luego de una intervención de parte del investigador haciendo que el estudiante observará las características del punto que dividía a cada intervalo.

Tarea 4.2. El objetivo de esta actividad es que mediante la visualización de los puntos medios que se van formando a partir de un intervalo inicial (como proceso iterativo de división en dos partes iguales del cada intervalo) estén en capacidad de describir con sus palabras lo que se establece en el Método de Bisección. Para esto se esperaba que los estudiantes den un valor 
estimado para imaginándose la gráfica de la función; también se espera que los estudiantes detallen paso a paso lo que se realizó en esta actividad, teniendo en cuenta los puntos medios y los intervalos que seleccionó. Se espera que los estudiantes argumenten que primero se tuvo un intervalo en donde la gráfica de la función que pasaba por estos puntos se interceptaba con el eje, luego con el punto medio de este intervalo se forman dos subintervalos y se toma el intervalo que conserva la intercepción y así sucesivamente con las demás divisiones de los intervalos.

Con estas actividades se cumplió el objetivo de hacer un primer acercamiento al Teorema de Bolzano mediante la visualización de los puntos medios que se van generando a partir de un intervalo inicial. Los estudiantes identifican que para uno de los dos nuevos intervalos que se van formando, se sigue conservando la intercepción entre la gráfica y el eje como se puede observar en los análisis antes mostrados.

También se alcanzó el objetivo de aproximar a los estudiantes al Método de Bisección mediante la visualización de los puntos medios que se van formando a partir de un intervalo inicial. Los estudiantes interpretan este proceso repetitivo y están en la capacidad de describir con sus palabras lo que se establece en este método, para el cual dan un valor aproximado de la intersección de la función con el eje y describen los pasos a seguir para encontrar este valor.

\section{CONCLUSIONES}

Dentro de los hallazgos de esta investigación se encontró que ambos estudiantes utilizaron técnicas similares en el desarrollo de las tareas, tal como se puede observar en la identificación de la variación de temperatura al manipular la perilla del termómetro y que esto ocurre al pasar por cero $0^{\circ}$, lo cual lleva a los estudiantes a verificar que no es posible pasar de una temperatura a otra sin tener un punto medio entre ellas $\left(0^{\circ}\right)$, análogamente sucede lo mismo en el llamado "punto de corte" en el eje de las abscisas, es decir donde existen las llamadas raíces y que estas solo ocurren cuando una función es continua y las imágenes de los extremos del intervalo en el que se encuentra son contrarias.

Cabe aclarar que ambos estudiantes identificaron que no necesariamente los puntos deben estar en diferentes cuadrantes para que exista una raíz, por lo que no es suficiente mirar la posición del intervalo inicial, pues cabe la posibilidad de existencia de una o más raíces en sus subintervalos; las cuales pueden variar de posición en los subintervalos dependiendo de los signos de las componentes, seguidamente se observa acompañada de la técnica identificación de signos de las componentes, el trazado de la función con el dedo o cursor, un recurso que utilizaron ambos estudiantes para confirmar si de verdad había una intercepción y en dónde ocurría esta. Como se ha relatado, los estudiantes tuvieron una apropiación respecto a cómo identificar la existencia de una raíz de una función en un intervalo dado; donde uno de ellos logró alcanzar los objetivos apropiándose del paso a paso para la implementación del método de bisección y la identificación de la raíz utilizando el teorema de Bolzano.

En cuanto a la implementación de las Situaciones A-didácticas para el acercamiento a las soluciones aproximadas de las ecuaciones no lineales, se lograron aplicar las actividades a la muestra de estudiantes de la institución, con resultados satisfactorios, pues se demostró que lograron adaptarse al medio que se les imponía (actividades mediante el software GeoGebra) sin la constante intervención del docente, mostrando así que los estudiantes por sí mismos, buscaran técnicas para el desarrollo de las tareas planteadas, las cuales se fueron consolidando en el transcurso de las actividades, atendiendo de esta manera las conclusiones y 
recomendaciones expuestas de Ochoa y Osorio (2017).

En general se puede concluir que Situaciones A-Didácticas cumplieron con su objetivo de acercar al estudiante al Método de Bisección, descubriendo (mediante la entrevista clínica) en los estudiantes las estrategias utilizadas a los largo de las actividades para generar sus propia conclusiones y generar hipótesis y conjeturas de lo que es este método de solución de ecuaciones no lineales (Hunting, 1997); Zazkis y Hazzan, 1999); Clement, 2000), a lo que se le es llamado construcción del conocimiento, por todo esto se garantizó en los estudiantes el aprendizaje por adaptación (Brousseau, 1997; 2007), en el cual ellos interactuaban con un medio, en este caso el medio fue virtual, en el cual ellos ejercían una acción este generaba una retroacción, la cual era interpretada por los estudiantes, para luego llegar a la validación.

\section{REFERENCIAS BIBLIOGRÁFICAS}

Acosta, G. M. (2010). Enseñando transformaciones geométricas con software de geometría dinámica Enseñando transformaciones geométricas con software de geometría dinámica. 132-142. Bogota D.C.: Cengage learning

Allan, C., Parra, S, y Martins, A. (2017) "Objetos de Aprendizaje para la Interpretación Geométrica de Métodos Numéricos: Uso de GeoGebra" Revista Iberoamericana de Educación en Tecnología y Tecnología en Educación, 20, 51-56.

Arias, F. (2012). El Proyecto de Investigación, 6ta edición. Caracas, Venezuela: Episteme Editorial.
Artigue, M., Douady, R., y Moreno, L. (1995). Ingeniería didáctica en educación matemática. Bogotá: Grupo Editorial Iberoamericana.

Schnettler, B. y Baer A. (2009) Hacia una metodología cualitativa audiovisual. El video como instrumento de investigación social in: A., Merlino (ed.), Investigación cualitativa en ciencias sociales: Temas, problemas y aplicaciones, Buenos Aires: Cengage Learning, 149-173.

Berrío, J. (2016). Estudio De La Construcción De Pasos De Razonamiento En El Proceso De Justificación Teórica En La Resolución De Problemas De Geometría [Trabajo de Grado de Maestría, Universidad Industrial de Santander, Bucaramanga].

Berrio, J.; Fiallo, J., y Acosta, M. (2013). Estudio de la construcción de pasos de razonamiento en el proceso de justificación teórica en la resolución de problemas de geometría. VII Congreso Iberoamericano de Educación Matemática, (1), 1611-1615.

Brousseau, G. (1997). Theory of Didactical Situations in Mathematics. Kluwer Academic Publishers.

Brousseau, G. (2007). Iniciacion al estudio de las teorias de las situaciones. Buenos Aires: Libros del Zorzal.

Calderon, O. y Cantillo, S. (2018). Situaciones Didácticas Mediadas Con Geogebra Para La Enseñanza Del Método De Bisección En Once Grado (Trabajo de grado de pregrado). Universidad del Atlántico, Barranquilla, Colombia.

Camargo, L. (2019). Estrategias cualitativas de investigación en Educación Matemática. XV Conferencia interamericana 
de Educación Matemática. https:// conferencia.ciaemredumate.org/ index.php/xvciaem/xv/paper/ viewFile/1061/568

Canale, R. y Chapra, S. (2014). Numerical Methods for Engineers. India: McGrawHill

Cervantes-Barraza, J., Berrío-Valbuena, J., Contreras-Vásquez, M., y MartínezFontalvo, V. (2021). Espacios de trabajo geométrico personal de profesores de matemáticas en formación. Educación y Humanismo, 23(40), 1-17. https://doi. org/10.17081/eduhum.23.40.4083

Chevallard, Y. (1998). La trasposición didáctica: del saber sabio al saber enseñado. Buenos Aires: Aiqué Grupo Editor.

Clement, J. (2000). Analysis of clinical interviews: Foundations and model viability. ( $R$. Lesh, \& A. Kelly, Edits.) Handbook of research methodologies for science and mathematics education, 341.385.

García, M. (2011). El vídeo como herramienta de investigación. Una propuesta metodológica para la formación de profesionales en Comunicación. Revista del CES Felipe II, 13(7), 1-12.

Hunting, R. (1997). Clinical interview methods in mathematics education research and practice. Journal of Mathematical Behavior, 16(2), 145-164.

Mora, W. F. (2018). Introducción a los métodos númericos. Costa Rica: Revista dígital, Matemática, Educación e Internet.

Ochoa, J., y Osorio, A. (2017). Ingeniería didáctica para el estudio del acercamiento de estudiantes con capacidades excepcionales a las soluciones aproximadas de ecuaciones no lineales (Trabajo de grado de pregrado). Universidad del Atlántico, Barranquilla, Colombia.

Hernández, R., Fernández, C. y Batista, P. (2014). Metodología de la Investigación. Ciudad de México: Mc Graw Hill Education.

Sánchez, M., y Revuelta, F. (2005). El proceso de transcripción en el marco de la metodología de investigación cualitativa actual. Enseñanza, (23), 367-386.

Valbuena-Duarte, S., De La Hoz, K., \& BerrioValbuena, J. (2021). El rol del docente de matemáticas en el desarrollo del pensamiento crítico en la enseñanza remota. Revista Boletín Redipe, 10(1), 372-386. https://doi.org/10.36260/rbr. v10i1.1188

Zazkis, R. \& Hazzan, O. (1999). Interviewing in mathematics education research: Choosing the questions. Journal of Mathematical Behavior, 17(4), 429-439. 